\title{
Plagiaterkennung und Plagiatvermeidung an Universitäten und Bibliotheken
}

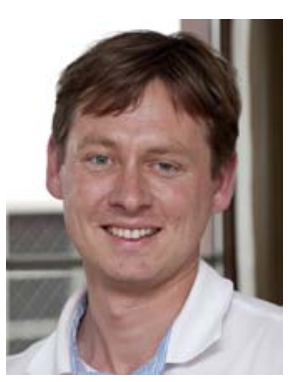

\section{Dr. Martin Nissen}

Universitätsbibliothek Heidelberg

Plöck 107-109

D-69117 Heidelberg

E-Mail: nissen@ub.uni-heidelberg.de

Trotz des großen öffentlichen Interesses und der hohen Relevanz bei der Bewertung von Prüfungsleistungen wurde das Thema Plagiaterkennung und Plagiatvermeidung von wissenschaftlichen Bibliotheken in Deutschland bisher nicht systematisch aufgegriffen. Den vorliegenden Untersuchungen zufolge wird die Häufigkeit von Plagiaten zukünftig weiter ansteigen. Ausgehend von der Beschreibung des Ist-Zustandes an Universitäten und Bibliotheken zeigt der Beitrag Möglichkeiten eines systematischen Umgangs mit Plagiaten als Form wissenschaftlichen Fehlverhaltens auf. Besondere Bedeutung kommt dabei Veranstaltungen zur Vermittlung von Informationskompetenz zu, die durch den Einsatz von Plagiaterkennungssystemen flankiert werden können. Ergänzt werden diese strategischen Überlegungen durch die Beschreibung konkreter Aktivitäten an der Universitätsbibliothek Heidelberg.

Schlüsselwörter: Informationskompetenz; Plagiat; Anwendungssoftware

\section{Recognition and Prevention of Plagiarism in Universities and Libraries: State of the Art and Strategic Considerations}

Despite of a significant public interest and a high relevance for the assessment of examination the recognition and prevention of plagiarism has not been sufficiently discussed by libraries in Germany. According to previous studies, the frequency of plagiarism will rise within the next years. After describing the current state at universities and libraries the article discusses different ways of a systematic approach to plagiarism as a form of scientific misconduct. Of high importance are classes in information literacy, which can be supported by the use of plagiarism detection systems. Besides these strategic considerations, the article decribes concrete activities at the University Library Heidelberg.

Keywords: Information literacy; plagiarism; application software

\section{Einleitung}

Durch die Plagiatsfälle des Jahres 2011 ist das Plagiat als Form wissenschaftlichen Fehlverhaltens verstärkt ins öffentliche Bewusstsein gerückt. Im Zentrum des Interesses standen die Doktorarbeiten von Politikerinnen und Politikern wie Karl-Theodor zu Guttenberg, Silvana Koch-Mehrin, Jorgo Chatzimarkakis und Veronica Saß. Die Plagiatsprüfung führte in diesen Fällen zur Aberkennung der Doktortitel. Die Universitäten reagierten mit einer Verschärfung der Promotionsordnung, der Aufnahme eidesstattlicher Erklärungen in das Promotionsverfahren sowie mit der Lizenzierung von Plagiaterkennungssoftware. Diskutiert wird seitdem die hohe Zahl von Promotionen insbesondere in den Massenfächern Jura, Medizin und Wirtschaftswissenschaften, die inflationäre Vergabe von Spitzennoten sowie die teilweise ungenügende Betreuung der Promovierenden durch ihre Professoren. ${ }^{1}$ Demzufolge betrifft das Thema Plagiat in der Wissenschaft vor allem Doktorarbeiten, bei denen absichtlich getäuscht wird, um den Arbeitsaufwand zu reduzieren und die Note zu verbessern.

Untersuchungen zur Häufigkeit von Plagiaten, die überwiegend auf anonymisierten Umfragen beruhen, kommen jedoch zu einem anderen Ergebnis. Danach nimmt die Häufigkeit von Plagiaten ab, je qualifizierter der Verfasser eines Textes ist. ${ }^{2}$ Doktoranden scheinen seltener zu plagiieren als jüngere Studenten. Zudem sind die Hauptgründe für Plagiate Überforderung, Unkenntnis wissenschaftlicher Arbeitstechniken und ein sich wandelndes Urheberrechtsverständnis, demzufolge Internetquellen nicht in gleicher Weise wie gedruckte Quellen zitiert werden müssen. ${ }^{3}$ Die absichtliche Täuschung in der Doktorarbeit eines extern Promovierenden stellt einen Sonderfall dar, der in seiner Bedeutung hinter die Vielzahl an unkorrekten Textübernahmen in Studienarbeiten zurücktritt.

Das Plagiat widerspricht fundamental den „Vorschlägen zur Sicherung guter wissenschaftlicher Praxis“, die von der Deutschen Forschungsgemeinschaft 1998 in einer Denkschrift formuliert und auf Universitätsebene in zahlreichen Satzungen umgesetzt wurden. ${ }^{4}$ Es verletzt nicht nur das Urheberrecht, sondern steht der Ausprägung einer eigenständigen wissenschaftlichen Arbeitsweise entgegen. Die eindeutige Kennzeichnung der Urheberschaft eines Textes ist eine wissenschaftsgeschichtliche Errungenschaft, die auch im digitalen Zeitalter nicht aufgegeben werden sollte.

1 Zuletzt: Friedmann, Jan \& Trenkamp, Oliver: Titel fürs Türschild. In: Der Spiegel 2012/9, S. 34-36.

2 Szabo, Attila \& Underwood, Jean: Cybercheats. Is Information and Communication Technology fuelling academic dishonesty? In: Active Learning in Higher Communication 5/2 (2004) S. 180-199.

3 Ercegovac, Zorana \& Richardson Jr., John V.: Academic Dishonesty, Plagiarism Included, in the Digital Age. A Literature Review. In: College and Research Libraries 65/4 (2004) S. 301-318, hier 306. Online: http://www.baruch.cuny.edu/facultyhandbook/documents/ PlagiarismLiteratureReview.pdfEvecovic.

4 Deutsche Forschungsgemeinschaft (DFG): Vorschläge zur Sicherung guter wissenschaftlicher Praxis. Empfehlungen der Kommission „Selbstkontrolle in der Wissenschaft“. Weinheim 1998. Online http:// www.dfg.de/download/pdf/dfg_im_profil/reden_stellungnahmen/ download/empfehlung_wiss_praxis_0198.pdf. 
Universitäten und Bibliotheken bieten sich drei Möglichkeiten, das Ansteigen der Häufigkeit von Plagiaten zu verhindern. Zentral ist erstens die Vorbildfunktion der guten wissenschaftlichen Praxis in Forschung und Lehre, zu der eine gute Themenstellung sowie ein enges Betreuungsverhältnis gehört. Zweitens sind Lehrveranstaltungen im Bereich der Vermittlung von Informationskompetenz geeignet, wie sie von Universitäten und Bibliotheken angeboten werden. Ergänzt wird der Umgang mit Plagiaten drittens durch die Entwicklung oder Lizenzierung von Plagiaterkennungssystemen, die insbesondere bei Verdachtsfällen eingesetzt werden sollten.

\section{Das Plagiat: Definition}

Unter einem Plagiat versteht man die Ausweisung fremden geistigen Eigentums als eigenes geistiges Eigentum. Der Duden spricht von der ,unrechtmäßige[n] Aneignung von Gedanken, Ideen o. Ä. eines anderen auf künstlerischem oder wissenschaftlichem Gebiet und ihre[r] Veröffentlichung; Diebstahl geistigen Eigentums ${ }^{\text {“5 }}$, wobei verwandte Begriffe wie Fälschung, Imitation oder Kopie abgegrenzt werden müssen. Unterscheiden lässt sich eine enge und eine weite Definition des Begriffs. Eng gefasst, bedeutet ein Plagiat die wortwörtliche Ausweisung fremden geistigen Eigentums als eigenes. Weiter gefasst, tritt die unzulässige Auslegung der urheberrechtlichen Schrankenregelung des Zitats (§ 51, UrhG) hinzu, bei der das Zitatrecht überdehnt, die wirkliche Urheberschaft verschleiert oder notwendige Verweise verkürzt werden.

Die Typologie des Plagiats ist vielfältig und reicht von unveränderten Übernahmen (Totalplagiat) über Teil-, Ideen-, Übersetzungs- und Strukturplagiate bis hin zu speziellen Techniken des Plagiierens wie der Verschleierung, dem unzulänglichen Zitat oder dem sogenannten Bauernopfer, bei dem ein Nachweis auf die zitierte Quelle an einer unbedeutenden Stelle dazu verwendet wird, die Quelle in der Folge ohne weitere Nachweise zu zitieren. ${ }^{6}$ In den vorliegenden Arbeiten von Debora Weber-Wulff, Professorin für Media and Computing an der Hochschule für Technik und Wirtschaft Berlin und führende Expertin für das Thema Plagiat und Plagiaterkennung in Deutschland, werden die verschiedenen Plagiatstypen umfänglich beschrieben. ${ }^{7}$

In welchem Umfang fremdes geistiges Eigentum ohne erforderlichen Quellenbeleg übernommen werden muss, damit die ganze Arbeit als Plagiat gilt, ist umstritten und je nach wissenschaftlicher Disziplin unterschiedlich. Von entscheidender Bedeutung ist dabei, wie wichtig die ungekennzeichnet übernommene Stelle für die eigene Argumentation

5 Der Duden Online: Plagiat, das. Online verfügbar unter: http://www. duden.de/rechtschreibung/Plagiat.

6 Stegemann-Boehl, Stefanie: Fehlverhalten von Forschern. Eine Untersuchung am Beispiel der biomedizinischen Forschung im Rechtsvergleich USA-Deutschland. Stuttgart 1994, S. 113 f.

7 Weber-Wulff, Debora \& Wohnsdorf, Gabriele: Strategien der Plagiatsbekämpfung. In: Information - Wissenschaft \& Praxis 57/2 (2006) S. $90-98$ ist, so dass bei entsprechender Bedeutung einer einzigen plagiierten Textstelle die gesamte Arbeit als Plagiat eingestuft werden kann.

Auch wenn das Plagiat kein juristischer Begriff ist und es in Deutschland keine gesetzliche Definition gibt, können die Folgen gravierend sein. Sie reichen von der Exmatrikulation bis zur Aberkennung akademischer Titel ${ }^{8}$ Die Frage, ob das Plagiat vorsätzlich oder unabsichtlich erfolgte, ist in der deutschen Rechtsprechung zunehmend ohne Belang. Klagen gegen Sanktionen wie die Aberkennung akademischer Grade blieben überwiegend erfolglos. ${ }^{9}$

\section{Umfrageergebnisse zur Häufigkeit von Plagiaten}

In den USA und Großbritannien wurden seit den 1990er Jahren Umfragen zur Häufigkeit von Wissenschaftsplagiaten durchgeführt. ${ }^{10}$ In einer Studie von Donald McCabe (2005), bei der ca. 73.000 Studierende an US-Colleges und Universitäten befragt wurden, gaben ca. ein Drittel der Befragten Plagiate in geringem Umfang zu, 7 Prozent übernahmen auch längere Textpassagen. ${ }^{11}$ Dabei lag die Quote bei Plagiaten aus gedruckten Quellen mit 38 Prozent leicht über der Quote aus Internet-Quellen (36 Prozent), was die These vom Internetplagiat relativiert. Eine britische Umfrage von 2006 mit einer Beteiligungszahl von ca. 1.000 Studierenden kam bei einem Anteil von ca. einem Drittel der Befragten, die zugaben, Ideen ungekennzeichnet zu übernehmen, zu ähnlichen Ergebnissen. ${ }^{12}$ Aus neueren US-Studien, die im Vergleich zu McCabe allerdings geringere Probandenzahlen aufweisen, geht hervor, dass die Zahl der Studierenden, die kürzere Textplagiate zugibt, seit den 1980er Jahren stark angestiegen ist. ${ }^{13}$ Insgesamt liegt die Häufigkeit von Plagiaten in wissenschaftlichen Studienarbeiten mit Werten zwischen 20 Prozent und 60 Prozent in neueren Umfragen sehr hoch. ${ }^{14}$

Neben der Häufigkeit geben die Umfragen auch Aufschluss über die Gründe für die Übernahme fremder Texte. Angeführt werden Leistungsdruck und Zeitmangel im Stu-

8 Vgl. Rieble, Volker: Das Wissenschaftsplagiat. Vom Versagen eines Systems. Frankfurt/Main 2010, S. 10. Besonders streng sind die Regelungen in den Bundesländern Baden-Württemberg und NordrheinWestfalen. Das Landeshochschulgesetz Baden Württemberg etwa benennt in der Fassung vom März 2009 das Anfertigen eines Plagiats explizit als Exmatrikulationsgrund.

9 Rieble (Anm. 8) S. 13.

10 Ein Überblick über die Forschungsliteratur bieten: Ercegovac (Anm. 3).

11 McCabe, Donald L.: Cheating among college and university students. A North American perspective. In: International Journal for Educational Integrity, 1/1 (2005) S. 1-11, hier 6.

12 Vgl. Weber, Stefan: Das Google-Copy-Paste-Syndrom. Wie Netzplagiate Ausbildung und Wissen gefährden. 2. Aufl. Hannover 2009, S. 56.

13 Jones, Dorothy L. R.: Academic Dishonesty. Are morge students cheating? In: Business Communication Quarterly 74 (2011) S. 141-150; Lehman, Carol M. \& DuFrene, Debbie: Business communication. 16. Aufl. Mason, OH 2011, S. 328; Howard, Rebecca Moore \& Davies, Laura J.: In: Educational Leadership 66/6 (2009) S. 64-67.

14 Vgl.: Kohl, Kerstin Eleonora: Geschummelt wird selten. Erfahrungen mit der „Freiwilligen Plagiatskontrolle“ für Studierende. In: Zeitschrift für Hochschulentwicklung 6/2 (2011) S. 160. 
dium, daneben jedoch auch eine generelle Unsicherheit beim wissenschaftlichen Arbeiten, die von der inhaltlichen Überforderung bis zur schlichten Unwissenheit reicht. ${ }^{15}$ So sind einer Umfrage von Dorothy Jones von 2011 zufolge lediglich 75 Prozent der Befragten der Ansicht, dass Internetquellen ebenfalls korrekt zitiert werden müssten und dass der Kauf eines Textes bei einem Internetanbieter nicht zur ungekennzeichneten Übernahme dieses Textes berechtigt. ${ }^{16}$

In den USA sind verschiedene Maßnehmen ergriffen worden, um die Häufigkeit von Plagiaten zu reduzieren. Dies umfasst den flächendeckenden Einsatz von Plagiaterkennungssystemen, die Einrichtung von Beratungszentren sowie die Gründung von Honor Boards, in denen bei Plagiatsfällen Studierende selbst die Sanktionen aussprechen. Als erfolgreich hat sich auch die Unterzeichnung sogenannter Ehrencodices erwiesen, in denen Studenten schriftlich zu Studienbeginn bzw. vor der Anfertigung einer schriftlichen Arbeit versprechen, wissenschaftlich redlich zu arbeiten. Der Erfolg dieser Anstrengungen wurde in begleitenden Studien empirisch belegt. ${ }^{17}$

In Deutschland ist die Plagiatsforschung bisher kein eigenständiges Forschungsfeld. Untersuchungen zu Form und Häufigkeit von Plagiaten sowie zu den rechtlichen und wissenschaftsethischen Grundlagen erfolgten insbesondere in den Rechts-, Bildungs- und Informationswissenschaften. ${ }^{18}$ Der Anteil von einem Drittel unter den Studierenden, die zumindest gelegentlich Textpassagen ungekennzeichnet übernehmen, scheint den vorliegenden Studien zufolge auch für den deutschen Raum zu gelten. ${ }^{19}$ Die prinzipielle Bereitschaft zu plagiieren, liegt einzelnen Studien zufolge mit rund 90 Prozent weit darüber, wohingegen Vollplagiate, d.h. die Übernahme ganzer Texte, selten sind. ${ }^{20}$ Kritisch zu bewerten sind Studien, an denen Unternehmen beteiligt waren, die Systeme zum Aufdecken von Plagiaten bereitstellen. In einer derartigen Studie von 2008, bei der ca. 600 Studierende einer Fachhochschule mit wirtschaftswissenschaftlichem Schwerpunkt interviewt wurden, gaben ca. 25 Prozent an, auch bei wortwörtlichen Zitaten nur selten die Quellen anzuführen. ${ }^{21}$ Für genauere Zahlen sind weitere Untersuchungen mit höheren Probandenzahlen erforderlich.

15 Für die USA: Synder, L. G., \& Cannoy, S. D.: Business communication. Instructional strategies to prevent plagiarism. In: Business Education Forum 65/1 (2010) S. 23-25. Zur Situation an der Pädagogischen Hochschule Freiburg vgl. exemplarisch: Kohl (Anm. 14) S. 161.

16 Jones (Anm. 13) S. 145.

17 Sattler, Sebastian: Unterschätztes Phänomen? Über den Umfang und Umgang mit Plagiaten. In: Forschung \& Lehre $15 / 5$ (2008), S. 298-299.

18 Ein Überblick über die Forschungsliteratur zum deutschsprachigen Raum findet sich bei: Kohl, Kerstin Eleonora: E-Plagiate und internetbasierte Plagiatsdetektion als didaktische Herausforderung und wissenschaftliche Chance. Inkorrekte Intertextualität in Texten Studierender. In: Ludwigsburger Beiträge zur Medienpädagogik, 14 (2011) S. 4. Online http://www.ph-ludwigsburg.de/2081.html.

19 Vgl. Sühl-Strohmenger, Wilfried: Wissensklau, Unvermögen oder Paradigmenwechsel. In. B.I.T. online 2009/4, S. 431-435.

20 Sattler (Anm. 17) S. 298.

21 Die Benutzung des Internets im Hochschulwesen. „Plagiat in Studienarbeiten“ (Juni 2008). Online: http://www.compilatio.net/uploads/ f88b37430244afb604cd2eaa49721 cfc/fichiers/080604-sixdegres-umfrage-plagiat-deutschland.pdf. Die Umfrage wurde durchgeführt in Zusammenarbeit der Unternehmen Six Degrees, compilatio.net und Sphinx Development.
Wichtigster Grund für die wachsende Häufigkeit von Plagiaten ist die Verfügbarkeit elektronischer Quellen im Internet, inklusive kommerzieller Angebote vollständiger Referate und wissenschaftlicher Arbeiten auf Plattformen wie referate.de, hausarbeiten.de und diplom.de. ${ }^{22}$ Für den deutschen Wissenschaftsbetrieb tritt die Umstellung auf die Bachelor-/Master-Studiengänge hinzu mit einem Zeit- und Prüfungsdruck und einer Zunahme von Essays als Prüfungsleistungen, die thematisch weniger stark spezialisiert sind und dadurch leichter aus vorhandenen Internetquellen zusammengesetzt werden können. ${ }^{23}$ Insgesamt zeigt sich, dass bei Studierenden mangelnde Schreibkompetenzen bestehen, die die Ausweisung fremder Texte als eigene begünstigen. ${ }^{24}$ Dies ist auch das Ergebnis eines Projekts von 2009/2010 an der Pädagogischen Hochschule Freiburg, bei dem den Studierenden im Rahmen einer Freiwilligen Plagiatskontrolle die Möglichkeit angeboten wurde, die eigenen Texte anonym mit Hilfe eines Plagiaterkennungssystens zu testen und die Ergebnisse in einer persönlichen Beratung zu besprechen. ${ }^{25}$ Der Test von rund 500 Arbeiten ergab, dass der Anteil von Arbeiten mit nicht korrekten oder fehlenden Zitierungen im Verlauf des Studiums zwar abnimmt - bei Studierenden aus den ersten drei Semestern waren 90 Prozent der Arbeiten fehlerhaft - mit rund 40 Prozent nicht korrekten oder fehlenden Zitierungen unter den Arbeiten Studierender höherer Semester (ab 6. Semester) liegt er jedoch noch immer sehr hoch. ${ }^{26}$

\section{Zum Umgang mit Plagiaten an Universitäten und Bibliotheken}

Die Digitalisierung der Arbeitstechniken, die wachsende Zahl verfügbarer Internetquellen, ein gewandeltes Urheberrechtsverständnis sowie die veränderte Studienkultur im Bologna-Zeitalter machen ein weiteres Ansteigen der Zahl von Plagiaten wahrscheinlich. Zwar wird den bisherigen Untersuchungen zufolge überwiegend aufgrund von Unkenntnis bzw. Inkompetenz plagiiert, zugleich entwickelt sich jedoch in den USA ein Markt für Tutorials, die in das absichtliche Täuschen einführen und fortgeschrittene Techniken des Plagiierens wie Übersetzungsplagiate sowie Neu- und Umformulierungen vermitteln (sogenanntes ,high-tech cheating $\left.{ }^{6 *}\right){ }^{27}$

Auch wenn die US-amerikanische Situation nicht eins zu eins übertragen werden kann, stellt der Umgang mit Plagiaten für die deutschen Hochschulen eine bisher wenig be-

22 Die Website www.hausarbeiten.de bietet ca. 130.000 Arbeiten zum kostenpflichtigen Download an (ca. 3-12 EUR pro Arbeit). Auf der Seite www.diplom.de sind ca. 13.000 Abschlussarbeiten verfügbar.

23 Sattler (Anm. 17).

24 Steinhoff, Torsten: Wissenschaftliche Textkompetenz. Sprachgebrauch und Schreibentwicklung in wissenschaftlichen Texten von Studenten und Experten. Tübingen 2007; Pohl, Thorsten: Studien zur Ontogenese wissenschaftlichen Schreibens. Tübingen 2007.

25 Vgl. Kohl (Anm. 14).

26 Kohl (Anm. 14) S. 167.

27 Seitz, Christopher M., Orsini, Mushin M. \& Gringle, Meredith R.: YouTube. An International Platform for Sharing Methods of Cheating. In: International Journal for Educational Integrity 7/1 (2011). Online http://www.ojs.unisa.edu.au/index.php/IJEI/article/view/744. 
achtete Herausforderung dar. Sehr kritisch hat sich hier der Rechtswissenschaftler Volker Rieble geäußert, der für das deutsche Wissenschaftssystem von einem ,institutionellen Versagen“" spricht, da das Phänomen von Verlagen, Universitäten und Wissenschaftsinstitutionen nicht ausreichend thematisiert würde. ${ }^{28}$

Im Umgang mit Plagiaten bieten sich Hochschulen und Bibliotheken drei Möglichkeiten, die sich wechselseitig ergänzen: Vermeidung von Plagiaten durch wissenschaftliche Exzellenz in Forschung und Lehre, Stärkung der wissenschaftlichen Arbeitstechniken durch Vermittlung von Informationskompetenz sowie der Einsatz von Plagiaterkennungssystemen zur Überprüfung von Plagiatsfällen.

Die Vorbildfunktion der Wissenschaft bleibt dabei zentral. Dies umfasst insbesondere die Rolle etablierter Fachvertreter bei der Ausbildung des wissenschaftlichen Nachwuchses, zu der auch die Betreuung von Qualifikationsarbeiten gehört. Ein gut gewähltes Thema, das die Balance von wissenschaftlicher Innovation und pragmatischer Handhabung wahrt, ist der beste Schutz gegen eine plagiierte Arbeit. Studienarbeiten zu standardisierten Themen als Prüfungsleistungen im Rahmen größerer Veranstaltungen erhöhen die Wahrscheinlichkeit von Plagiaten hingegen beträchtlich.

\subsection{Prävention statt Kontrolle: Veranstaltungen zur Vermittlung von Informationskompetenz}

Unkorrekte oder fehlende Zitierungen in Studienarbeiten sind häufig nicht Teil einer gezielten Täuschungsstrategie, sondern Ergebnis eines inkompetenten Umgangs mit wissenschaftlicher Information. Insbesondere frei verfügbare Internetquellen verleiten Studierende dazu, diese zu übernehmen, ohne sie in erforderlicher Weise zu kennzeichnen. Gestützt wird dieses Vorgehen durch die Behauptung, Internetquellen, die wie beispielsweise Wikipedia-Artikel unter freie Lizenzen gestellt werden, beförderten den geistigen Diebstahl, auch wenn die weit verbreiteten Creative-CommonsLizenzen den Verweis auf den Urheber zwingend erfordern.

Zur Vermeidung von Plagiaten sind vermehrt Einführungen in das wissenschaftliche Arbeiten erforderlich, wie sie im Rahmen von Tutorien an den Universitäten sowie bei Veranstaltungen zur Vermittlung von Informationskompetenz an Bibliotheken angeboten werden. Als Teil der Vermittlung von Informationskompetenz ist das Thema Plagiat und Plagiaterkennung erst in jüngster Zeit verstärkt ins Blickfeld geraten. Dabei rechnete die Association of College and Research Libraries (ACRL) bereits im Jahr 2000 den verantwortungsvollen Umgang mit geistigem Eigentum zu den Information Literacy Competency Standards for Higher Education. ${ }^{29}$ Standard 5 beschreibt die Kompetenz, Informa-

28 Rieble (Anm. 8) S. 57.

29 The Association of College and Research Libraries: Information Literacy Competency Standards for Higher Education. Chicago, llinois 2000. Online verfügbar unter: http://www.ala.org/acrl/sites/ala.org. acrl/files/content/standards/standards.pdf. Zum Umgang mit Plagiaten als Teil der Vermittlung von Informationskompetenz vgl. auch: Schmid-Ruhe, Bernd: Mit Leichtigkeit zum Plagiat. Herausforderungen an Bibliotheken im Zeitalter der digitalen Wissenschaftskommu- tionen ethisch und rechtlich legal zu nutzen. Danach müsse der informationskompetente Student ein Verständnis dafür haben, "what constitutes plagiarism and does not represent work attributable to others as his/her own". ${ }^{30}$ Dies betrifft den Umgang mit gedruckten und elektronischen Quellen gleichermaßen.

Der rechtlich und ethisch korrekte Umgang mit wissenschaftlicher Information stellt an die Veranstaltungen zur Vermittlung von Informationskompetenz hohe Anforderungen. Sinnvoll ist es, das Thema Plagiat und Plagiatvermeidung in bereits bestehende Veranstaltungen zu integrieren. Dies umfasst zum einen Kurse, die in die wissenschaftlichen Arbeitstechniken einführen und Kompetenzen beim Nachweisen und Zitieren von wissenschaftlicher Literatur vermitteln. Vor allem bei der Zitation von Internetquellen bestehen den vorliegenden Studien zufolge große Unsicherheiten, gegen die in diesem Zusammenhang vorgegangen werden muss. Dies wird insbesondere im Rahmen von Tutorien geleistet, die sich an jüngere Studierende richten. Darüber hinaus sind fortgeschrittene Veranstaltungen zum Urheberrecht sowie Kurse zu den wissenschaftsethischen Grundlagen des Publikationswesens geeignet. Möglich wäre zudem der Aufbau von Beratungszentren nach dem Vorbild der britischen Plagiarism Advisory Services, die vom Joint Information Systems Commitee (JISC) gefördert werden. ${ }^{31}$ Der Plagiarism Advisory Service bietet nicht nur Beratung im Umgang mit dem Thema Plagiarismus an, sondern führt auch Studien und Umfragen durch, veranstaltet Tagungen und publiziert Forschungsberichte. An deutschen Hochschulen sind in diesem Bereich erste Planungen feststellbar. ${ }^{32}$

Bei dem Thema Plagiat und Plagiaterkennung arbeiten Bibliotheken und Universitäten eng zusammen. Über die Einbindung in wissenschaftliche Propädeutika hinaus bieten sich den Universitäten jedoch weitere Maßnahmen, die aufgrund der Plagiatsfälle des Jahres 2011 verschärft bzw. neu eingeführt wurden. Hierzu zählen die Einrichtung einer Kommission zum Umgang mit Fehlverhalten in der Wissenschaft, deren Arbeit von unabhängigen Ombudspersonen begleitet wird, die Einführung eidesstattlicher Erklärungen bei Qualifikationsarbeiten aller Stufen sowie die elektronische Abgabe von Studienarbeiten, die parallel zur gedruckten Ausgabe erfolgen kann.

Auch Plagiatsexperten, die den begleitenden Einsatz IT-gestützter Erkennungssysteme befürworten, sind sich einig, dass die Vermeidung von Plagiaten mehr Erfolg verspricht als Kontrolle und Abschreckung. Angesichts der

nikation. In: BuB: Forum Bibliothek und Information 60/3 (2008) S. 231-234.

30 The Association of College and Research Libraries (Anm. 29) S. 16. Deutsche Übersetzung: Homann, Benno: Standards der Informationskompetenz. Eine Übersetzung der amerikanischen Standards der ACRL1 als argumentative Hilfe zur Realisierung der „Teaching Library“. In: Bibliotheksdienst 36/5 (2002), S. 638. Online: http:// bibliotheksdienst.zlb.de/2002/02_05_07.pdf.

31 Duggan, Fionna: The Plagiarism Advisory Service. In: Library and Information Research 86/27 (2003) S. 37-42; Bernd Schmid-Ruhe (Anm. 29).

32 Eine Ausweitung der Beratungsangebote in diesem Bereich plant beispielsweise die Pädagogische Hochschule Freiburg: Kohl (Anm. 14). 
technischen Möglichkeiten erfordert der verantwortungsvolle Umgang mit wissenschaftlicher Information Kompetenzen, die über klassische wissenschaftliche Arbeitstechniken wie Zitat und Beleg hinausgehen. Zu denken ist hier insbesondere an urheberrechtliche Kompetenzen und einen kritischen Umgang mit der Authentizität digitaler Quellen. Durch technische Verfahren, die eine (halb-) automatisierte Übernahme fremder Quellen anhand individueller Filterfunktionen ermöglichen, wird die Anforderung an die Bewertung von Texten zukünftig weiter steigen. ${ }^{33}$

\subsection{Erkennen von Plagiaten mit und ohne Softwareeinsatz}

Das Aufdecken von Plagiaten ohne den Einsatz von Erkennungssystemen ist möglich, es stellt an den Prüfer jedoch hohe Anforderungen, die eine gute Kenntnis der Forschungsliteratur und ein genaues Verständnis für die Funktionsweise von Texten erfordern. Wichtige Indikatoren sind Stilbrüche, das gehäufte Auftreten von Fachtermini und Fremdworten sowie eine auffällige Kluft zwischen mündlicher und schriftlicher Leistung. Von eminenter Bedeutung ist hier die Betreuungssituation. Für einen Lehrer sind Stilbrüche und unerklärliche Leistungssteigerungen in schriftlichen Arbeiten leicht feststellbar. In Veranstaltungen mit hohen Teilnehmerzahlen an Universitäten, die als Prüfungsleistung eine abschließende schriftliche Arbeit erfordern, sind derartige Stil- und Leistungsbrüche hingegen kaum aufzudecken.

Als sehr erfolgreich bei der intellektuellen Aufdeckung von Plagiatsfällen haben sich die neuen kollaborativen Arbeitsweisen in Plag-Wikis erwiesen, in denen sich Nutzer zusammenschlossen, um die Doktorarbeiten insbesondere prominenter Politiker und Politikerinnen zu überprüfen. Bekanntestes Beispiel ist das GuttenPlag Wiki, bei dem sich über 1.000 Nutzer an dem Nachweis von Plagiaten in der Doktorarbeit des ehemaligen Verteidigungsministers KarlTheodor zu Guttenberg beteiligten. ${ }^{34}$ Auf der wichtigsten deutschen Plattform zur Plagiaterkennung im Internet vroniplag.wikia.com werden aktuell 20 Arbeiten auf die Häufigkeit von Plagiaten hin überprüft. ${ }^{35}$ Die Überprüfung umfasst jedoch ausschließlich Qualifikationsarbeiten prominenter Politiker und Wissenschaftler und ist in Anbetracht der aufwändigen Arbeitsweise für den Großteil der wissenschaftlichen Arbeiten ungeeignet. Insbesondere Studienarbeiten, bei denen den vorliegenden Untersuchungen zufolge von der höchsten Plagiatsdichte ausgegangen werden muss, lassen sich mithilfe von Plag-Wikis nicht überprüfen.

Größeren Erfolg verspricht hier der Einsatz sogenannter webbasierter Plagiaterkennungssysteme (PES), wie sie in den USA seit den 1990er Jahren eingesetzt werden und in Großbritannien seit etwa 10 Jahren weite Verbreitung ge-

33 Die Annotationen auf Begriffsebene, die diese Neustrukturierung des Textes ermöglichen, werden teilweise als Nano Publications bezeichnet: Mons, Barend \& Velterop, Jan: Nano-Publication in the e-science era (2009). Online: http://www.w3.org/wiki/images/4/4a/ HCLS\$ \$IWC2009\$\$orkshop\$Mons.pdf.

34 http://de.guttenplag.wikia.com/wiki/GuttenPlag_Wiki.

35 http://de.vroniplag.wikia.com/wiki/Home. funden haben. In Deutschland sind Plagiaterkennungssystem beispielsweise an den Universitäten Hamburg, Köln, Bielefeld, Hannover, Heidelberg sowie an der TU Dortmund im Einsatz. Bisher liegen allerdings kaum Campuslösungen, sondern überwiegend Lizenzierungen einzelner Institute oder Fakultäten vor. Größere Verbreitung haben IT-gestützte Formen der Plagiaterkennung in Österreich und der Schweiz gefunden. ${ }^{36}$

Die Leistungsfähigkeit der angebotenen Software sollte jedoch nicht überschätzt werden. ${ }^{37}$ Zum einen umfasst die Überprüfung bisher fast ausschließlich frei im Internet zugängliche Quellen. Der Abgleich mit lizenzpflichtigem Material, das den größten Teil der relevanten wissenschaftlichen Information umfasst, steht erst am Anfang, da nur wenige Anbieter begonnen haben, hierfür Vereinbarungen mit Verlagen abzuschließen. Darüber hinaus werden bisher Plagiate aus gedruckten Quellen, Ideen- und Strukturplagiate, Übersetzungsplagiate sowie nicht gekennzeichnete Paraphrasen nicht erkannt. Durch gezielte Manipulationen wie Umformulierungen, die Verwendung von Synonymen sowie das Vertauschen der Reihenfolge bei Aufzählungen lassen sich die Systeme leicht überlisten. Auch nimmt ein Erkennungssystem einem Prüfer die Arbeit nur zum Teil ab, da jede in den Prüfberichten markierte Stelle intellektuell bewertet werden muss. Je nach Einstellung werden von dem System auch korrekt zitierte Textstellen, bibliographische Angaben sowie Standardformulierungen markiert. Kritisiert wird teilweise auch die urheberrechtliche Grauzone, in der sich einige Systeme bewegen, sowie das gestörte Vertrauensverhältnis zwischen Verfasser und Gutachter, das eine maschinelle Überprüfung der Texte mit sich bringen kann.

Trotz der erwähnten Schwächen erscheint der Einsatz von Plagiaterkennungssystemen im Hochschulbetrieb sinnvoll. Flankierend zu den Veranstaltungen zur Vermittlung von Informationskompetenz kann die abschreckende Wirkung von Erkennungssoftware die Häufigkeit von Plagiaten reduzieren. Ferner ist eine händische maschinelle Überprüfung mit Hilfe von gängigen Suchmaschinen auf der Grundlage eines Anfangsverdachts angesichts der Vielzahl an Prüfungsarbeiten nicht zu bewältigen. Die Erkennungssoftware hilft, ein Verfahren, das weitgehend auch manuell durchgeführt werden könnte, zu beschleunigen und das Ergebnis grafisch aufzubereiten. Zuletzt ist davon auszugehen, dass sich in den nächsten Jahren die Aufdeckungsrate und der Umfang der überprüften Internetquellen beträchtlich erhöhen werden. Gerade in Deutschland sind bisher angesichts des noch immer kleinen Marktes erst wenige Anbieter aktiv geworden.

36 Sühl-Strohmenger (Anm. 18), S. 434.

37 Darauf weisen die von Debora Weber-Wulff durchgeführten Tests von Plagiaterkennungssoftware hin. http://plagiat.htw-berlin.de/software/. Weber-Wulff (Anm. 7); vgl. auch: Kramer, André: Plagiatoren ein Ende. Software und Web-Dienste kommen Plagiatoren auf die Schliche. In: c't 2011/15, S. 120-127. 


\subsection{Vorgehen an der Universitätsbibliothek Heidelberg}

Im Folgenden soll ein mögliches strategisches Vorgehen beispielhaft an den Aktivitäten der Universitätsbibliothek Heidelberg aufgezeigt werden. Die Universitätsbibliothek Heidelberg ist bei dem Thema Plagiaterkennung und Plagiatvermeidung in zwei Bereichen aktiv. Dies betrifft zum einen die Einbindung der Thematik in die Veranstaltungen zur Vermittlung von Informationskompetenz bzw. die Durchführung eigenständiger Workshops zur Plagiaterkennung, zum zweiten die Bereitstellung von Plagiaterkennungssoftware.

Seit dem Sommersemester 2011 bietet die Universitätsbibliothek Heidelberg regelmäßig Workshops zum Thema Plagiat und Plagiaterkennung an. Das Interesse an diesen Veranstaltungen wurde durch die Plagiatsfälle des Frühjahrs 2011 verstärkt, in Heidelberg namentlich durch die Überprüfung der Doktorarbeit der Europaabgeordneten Silvana Koch-Mehrin.

Die Workshops, die sich vorrangig an Mitarbeiterinnen und Mitarbeiter der Universität, die mit Prüfungsaufgaben betraut sind, richten, bieten ein interdisziplinäres Forum für die Diskussion zum Thema Fehlverhalten in der Wissenschaft. In dem ersten Teil werden die Umfrageergebnisse zur Häufigkeit von Plagiaten vorgestellt und rechtliche und wissenschaftspolitische Rahmenbedingungen aufgezeigt. Von besonderem Interesse bei der Bewertung wissenschaftlicher Texte sind dabei die Grenzen zwischen Zitat und Plagiat in den verschiedenen Wissenschaftsdisziplinen, wobei lediglich das Zitat im deutschen Urheberrecht gesetzlich definiert ist ( $\$ 51, \mathrm{UrhG})$. Anschließend haben die Teilnehmer die Möglichkeit, verschiedene Plagiaterkennungssysteme anhand selbst mitgebrachter Beispieltexte zu überprüfen. Auch hierbei zeigt sich, dass die bisherigen Systeme je nach verwendeter Quellenbasis in den verschiedenen Fachdisziplinen zu sehr unterschiedlichen Ergebnissen kommen. Ziel ist es, diese Veranstaltungen vor dem Hintergrund eines steigenden Bedarfs bei dieser Thematik zu verstetigen.

Über die Einbindung in Veranstaltungen zur Vermittlung von Informationskompetenz hinaus bietet die Universitätsbibliothek Heidelberg seit 2011 Mitgliedern der Universität die Möglichkeit, wissenschaftliche Texte mithilfe von Plagiaterkennungssoftware zu überprüfen. Nach mehreren Tests, bei denen Universitätsbibliothek und Rechenzentrum der Universität Heidelberg zusammenarbeiteten, ist seit Juni 2012 die Erkennungssoftware turnitin des amerikanischen Anbieters iParadigms im Einsatz. Diese bietet den Vorteil einer weltweit großen Verbreitung. Zudem kann das System als Lernplattform in die Lehre eingebunden werden.

\section{Zusammenfassung}

Das öffentliche Interesse an dem Thema Plagiat und Plagiaterkennung ist nach einem Höhepunkt im Frühjahr 2011 in den letzten Monaten zurückgegangen. Diese Entwicklung wurde durch die Fokussierung auf Doktorarbeiten der Geis- tes-, Sozial- und Wirtschaftswissenschaften verstärkt. Den vorliegenden Untersuchungen zufolge, die auf Umfragen unter Studierenden und Wissenschaftlern beruhen, ist jedoch von einer anhaltend hohen Zahl von Plagiaten auszugehen. Im größten Teil der Fälle können Plagiate aus Kapazitätsgründen nicht aufgedeckt werden.

Vier Gründe machen eine weiter steigende Zahl von Plagiaten wahrscheinlich: Erstens nimmt die Anzahl frei verfügbarer Internetquellen rapide zu und damit auch die Möglichkeit, diese für die eigene Arbeit ohne den erforderlichen Nachweis zu verwenden. Zweitens entstehen technische Verfahren, die eine automatisierte Übernahme fremder Texte anhand individueller Filterfunktionen ermöglichen. Drittens lässt sich ein gewandeltes Urheberrechtsverständnis feststellen, das eine scharfe Grenzziehung zwischen fremden und eigenen Texten zunehmend in Frage stellt. Zudem führt die Umstellung auf die Bachelor- / Master-Studiengänge viertens dazu, dass vermehrt Prüfungsleistungen im Rahmen von Massenveranstaltungen eingefordert werden, bei denen die Themenstellung nicht mehr in vergleichbarer Weise individuell abgestimmt werden kann.

Im Umgang mit Plagiaten haben die Hochschulen in Deutschland mehrere Möglichkeiten. Der beste Schutz liegt in der Vorbildfunktion wissenschaftlicher Exzellenz, bei der die Begeisterung über das Erreichen individueller Ziele im Mittelpunkt steht. Ferner verstärken Universitäten und Bibliotheken ihr Engagement bei der Vermittlung von Informationskompetenz. In diesen Veranstaltungen, die von Einführungen, Tutorien und Workshops bis zu Vorträgen zu Spezialthemen reichen und sich an sämtliche Qualifikationsstufen richten, können wissenschaftliche Arbeitstechniken erprobt und Kenntnisse zu rechtlichen und wissenschaftsethischen Fragestellungen vermittelt werden.

Die Vermittlung von Informationskompetenz sollte bei der Vermeidung von Plagiaten höher gewichtet werden als die abschreckende Wirkung von Plagiaterkennungssystemen. Dennoch kann angesichts der zu erwartend höheren Leistungsfähigkeit der Systeme der flankierende Einsatz von Erkennungssoftware hilfreich sein. Aktuell durchgeführten Pilotprojekten zufolge umfasst dies sowohl die Kontrolle bereits eingereichter Arbeiten als auch die Beratung zu wissenschaftlichem Fehlverhalten in Beratungszentren, die dem Vorbild der britischen Plagiarism Advisory Services folgend neu eingerichtet werden müssten.

Hilfreich beim Vorgehen gegen Plagiarismus und wissenschaftliches Fehlverhalten ist auch die steigende Anzahl von Publikationen, die im Sinne des Open Access über das Internet frei zugänglich und damit für den Nutzer leicht verfügbar sind. Zum einen wird der Quellennachweis bei schnellerer Verfügbarkeit vereinfacht, zudem vergrößert sich bei einer maschinellen Überprüfung der indexierte Bestand, mit dem der zu überprüfende Text abgeglichen werden muss. ${ }^{38}$ Wenn die Publikation elektronisch veröffentlicht wird und ein großer Teil der verwendeten Quellen über das Internet frei zugänglich ist, steigt die Gefahr, mit einem Plagiat tatsächlich aufzufallen. In dem Projekt Open Access Plagiat

38 Rieble (Anm. 8) S. 52; Sühl-Strohmenger (Anm. 20) S. 434. 
Suche (OAPS), das vom Institut für Betriebssysteme und Rechnerverbund der TU Braunschweig und dem Fachbereich Informationstechnologie der Physikalisch-Technischen Bundesanstalt (PTB) durchgeführt wird, werden Textdokumente auf Übereinstimmungen mit Open-Access-Dokumenten überprüft. ${ }^{39}$ Dabei deckt das Erkennungssystem nicht nur die ungekennzeichnete Übernahme fremden geistigen Eigen-

39 Brandt, Jens, Gutbrod, Martin, Wellnitz, Oliver \& Wolf, Lars: Plagiarism Detection in Open Access Publications. In: Tagungsband der vierten International Plagiarism Conference, Newcastle Upon Tyne 2010. Online http://oaps.eu/project/publication/4ipc-2010-brandt.pdf. tums auf, sondern weist auch die Mehrfachverwendung von Texten desgleichen Verfassers nach. Dadurch könnte auch der weit verbreiteten Kultur des Autoplagiats, die sowohl im Subskriptions- als auch im Open-Access-Modell hohe Kosten verursacht, ein Riegel vorgeschoben werden. ${ }^{40}$

Letzte Überprüfung der angeführten Online-Quellen: 10.06.2012.

40 Vgl. Müller Uwe: Open Access und die Kosten. In: BIBLIOTHEK Forschung und Praxis 35 (2011), S. 161-168. 